\title{
Interval inversion approach for an improved interpretation of well logs
}

\author{
Mihály Dobróka ${ }^{1}$, Norbert Péter Szabó ${ }^{2}$, József Tóth $^{3}$, and Péter Vass ${ }^{3}$
}

\begin{abstract}
The quality analysis of well-logging inversion results has always been an important part of formation evaluation. The precise calculation of hydrocarbon reserves requires the most accurate possible estimation of porosity, water saturation, and shale and rock-matrix volumes. The local inversion method conventionally used to predict the above model parameters depth by depth represents a marginally overdetermined inverse problem, which is rather sensitive to the uncertainty of observed data and limited in estimation accuracy. To reduce the harmful effect of data noise on the estimated model, we have suggested the interval inversion method, in which an increase of the overdetermination ratio allows a more accurate solution of the well-logging inverse problem. The interval inversion method inverts the data set of a longer depth interval to predict the vertical distributions of petrophysical parameters in a joint inversion procedure. In formulating the forward problem, we have extended the validity of probe response
\end{abstract}

functions to a greater depth interval assuming the petrophysical parameters are depth dependent, and then we expanded the model parameters into a series using the Legendre polynomials as basis functions for modeling inhomogeneous formations. We solved the inverse problem for a much smaller number of expansion coefficients than data to derive the petrophysical parameters in a stable overdetermined inversion procedure. The added advantage of the interval inversion method is that the layer thicknesses and suitably chosen zone parameters can be estimated automatically by the inversion procedure to refine the results of inverse and forward modeling. We have defined depth-dependent model covariance and correlation matrices to compare the quality of the local and interval inversion results. A detailed study using well logs measured from a Hungarian gas-bearing unconsolidated formation revealed that the greatly overdetermined interval inversion procedure can be effectively used in reducing the estimation errors in shaly sand formations, which may refine significantly the results of reserve calculation.

\section{INTRODUCTION}

In oilfield practice, we use open-hole logging data to determine essential petrophysical parameters of geologic formations that underlie the calculation of hydrocarbon reserves. Some reservoir parameters, such as effective porosity, water saturation, shale content, and fractional volume of rock matrices, can be determined with their estimation errors from the joint inversion of suitable well logs. In well $\log$ analysis, we assume known mathematical relations, called probe response functions, between the observations and the petrophysical model to predict data with the actual model. In the inversion procedure, one gives an estimate to the model parameters by fitting theoretical data to the measured data. Excellent overviews on the theory and computer implementation of well-logging inversion methods are given by Mayer and Sibbit (1980), Alberty and Hashmy (1984), and Ball et al. (1987).

Commercial inversion techniques routinely used in formation evaluation simultaneously process all the data collected with different probes from a certain depth to determine the petrophysical properties of reservoir rocks to the same depth. In shaly sand analysis, we usually have barely more types of probes than the number of unknowns in a given depth; thus, we have to solve a set of marginally overdetermined inverse problems along a borehole. Although the local inversion method gives fast and satisfactory results, its narrow overdetermination (data-to-unknowns ratio) sets a limit to estimation accuracy, which makes it a relatively noise-sensitive inversion procedure. The quality of estimation results can be im-

\footnotetext{
Manuscript received by the Editor 7 August 2015; revised manuscript received 5 October 2015; published online 22 February 2016.

${ }^{1}$ University of Miskolc, Department of Geophysics, Miskolc, Hungary. E-mail: dobroka@uni-miskolc.hu.

${ }^{2}$ University of Miskolc, Department of Geophysics, Miskolc, Hungary; MTA-ME, Geoengineering Research Group, Miskolc, Hungary. E-mail: norbert.szabo .phd@gmail.com.

${ }^{3}$ MOL Hungarian Oil and Gas Plc., Petrophysical Department, Szolnok, Hungary. E-mail: jtoth@mol.hu; pvass@mol.hu.

(C) 2016 Society of Exploration Geophysicists. All rights reserved.
} 
proved by increasing the amount of observed information using more types of well logs, which has well-known technological limits. There is another possibility to improve the accuracy of inversion estimates. We suggest increasing the overdetermination ratio of the inversion procedure by inverting the data set of a longer processing interval in a joint inversion procedure. Dobróka and Szabó (2001) introduced the interval inversion method that uses depth-dependent response functions (instead of local ones) in solving the forward problem. In the phase of inversion, we use a series expansion-based model discretization technique and invert at least an order (or orders) of magnitude higher number of data than unknowns compared with local inversion. The use of our inversion strategy bears great influence on the accuracy and reliability of model parameters extracted by inversion. Heidari et al. (2012) also emphasized the importance of inverting a data set collected from more depths jointly to improve the efficiency of well log interpretation.

Model discretization by means of series expansion is extensively used in natural and technical sciences, including geophysical research. In geoelectric data processing, the series expansion-based inversion methodology was used to evaluate the $2 \mathrm{D}$ resistivity and thickness distribution of shallow structures and an optimization method was suggested for giving the optimal number of inversion unknowns (Gyulai and Ormos, 1999). They show significant improvement in reducing the estimation errors and ambiguity. They also increase the lateral resolution without using any smoothness constraint. This geoelectric inversion algorithm is further developed for 3D applications by Gyulai and Tolnai (2012). A series expansion-based joint inversion method for the interpretation of DC geoelectric and seismic refraction data measured above $2 \mathrm{D}$ geologic structures is introduced by Kis (2002). In gravity modeling, the series expansion approach opened the door to reconstruct the $3 \mathrm{D}$ potential function and the full Eötvös-tensor from a large number of astronomical, torsional pendulum, and gravity data by solving a greatly overdetermined inverse problem (Dobróka and Völgyesi, 2008). Turai (2011) gives an estimate to the relaxation time spectrum using a series expansion-based inversion of induced polarization data. Dobróka et al. (2015) publish a series expansion-based Fourier transformation method in which the Fourier spectrum was developed in the framework of a significantly overdetermined inverse problem characterized by highly improved noise rejection capability.

The series expansion-based interval inversion method developed for well log analysis has been previously used to estimate the petrophysical parameters and thicknesses of homogeneous layers. To find the global minimum of misfit between observed and predicted data a simulated annealing (SA) optimization procedure was implemented, which showed considerable improvement in the suppression of data noise compared with local inversion procedures. The layer thicknesses cannot be extracted by any local inversion method because neither the local response functions include them explicitly nor the data measured at a given depth contain information on the position of distant layer boundaries. However, the basis functions of series expansion can be chosen as depending on the boundary coordinates, which allows the interval inversion procedure to estimate them automatically (Dobróka and Szabó, 2001). In addition, the lateral variation of formation boundaries along a profile of boreholes together with petrophysical parameters are determined from a 2D interval inversion procedure by Dobróka et al. (2009). An oilfield application for the estimation of layer- thicknesses using a float-encoded genetic algorithm is shown by Dobróka and Szabó (2012). Because the interval inversion method allows treating increasing number of unknowns without the significant decrease of overdetermination, one can estimate additional unknowns of interest. As a new feature of the interval inversion method, the simultaneous determination of conventional petrophysical (volumetric) properties and textural parameters, that is, cementation exponent, saturation exponent, and tortuosity factor, is suggested by Dobróka and Szabó (2011), whereas the evaluation of carbonate and metamorphic reservoirs is shown by Dobróka et al. (2012).

The advantages of the interval inversion method using a homogeneous layer approximation are represented by the optimal overdetermination ratio, accurate and reliable solution, effective noisesuppression feature, and automatic estimation of layer thicknesses. However, we also find some practical drawbacks, for instance, if we neglect the lithologic variations within inhomogeneous layers, we obtain only a rough estimation result typically indicated by high level of misfit between the predicted and observed data. As a consequence, the layerwise homogeneous approximation can result in poor vertical resolution in the evaluation of inhomogeneous formations. In this study, we introduce a further-developed interval inversion method using a Legendre polynomial-based discretization technique to increase the vertical resolution in inhomogeneous hydrocarbon-bearing formations. The use of the orthogonal set of basis functions decreases the data misfit compared with layer-by-layer inversion and assures reduced correlation between the model parameters. Largely increased overdetermination of the inverse problem results in a highly accurate estimation of the petrophysical model, whereas the usage of linear optimization provides a quick and steady convergence to the optimum. We compare the conventionally used local inversion procedure to the interval inversion method in a detailed study using real well-logging data. We show that the latter provides a significant improvement in the accuracy and reliability of inversion estimates, which has unequivocal practical benefits in the calculation of hydrocarbon reserves.

\section{THEORETICAL OVERVIEW}

\section{Local inversion method}

Let $\mathbf{d}^{\text {(obs) }}$ be an $S$-by-1 vector of well-logging data observed by different probes in a certain depth and $\mathbf{m}$ be an $P$-by- 1 vector of model parameters defined in the same depth. The former includes various nuclear, acoustic, and electric logs, whereas the latter incorporates the volumetric ratios of rock constituents as unknowns of the local inverse problem. In forward modeling, the sth data are calculated as follows:

$$
d_{s}^{(\mathrm{cal})}=g_{s}\left(m_{1}, m_{2}, \ldots, m_{P}, c\right),
$$

where $g_{s}$ represents the local response function of the $s$ th logging tool $(s=1, \ldots, S)$ and $S$ is the total number of probes. In the general case, the set of probe response functions is nonlinear, which may depend on all model parameters, $m$, and a set of zone parameters, $c$ such as the physical (well-log) properties of pore fluids and mineral components unvarying or just slowly varying in the hydrocarbon zone. By treating the zone parameters as a known constant, we solve a marginally overdetermined inverse problem in shaly sands, in which the number of data is barely more than that of 
the model parameters. We write the linear approximation of equation 1 in a subscripted form as follows:

$$
d_{s}^{(\mathrm{cal})}=\sum_{i=1}^{P} G_{s i} m_{i},
$$

where $G_{s i}=\left(\partial g_{s} / \partial m_{i}\right)_{m_{0}}$ is the element of Jacobi's matrix calculated in the $m_{0}$ point of the parameter space continuously improved during an iteration procedure. The objective function of the inverse problem to be minimized is

$$
E=\|\mathbf{e}\|_{2}^{2}+\varepsilon^{2}\|\mathbf{m}\|_{2}^{2},
$$

where e denotes the $S$-by-1 vector of deviations between the observed and calculated data normalized to the uncertainty of data $\varepsilon$ is a regularization parameter used for numerically stabilizing the solution of the inverse problem. If we do not have prior information on data variances, the residual errors can be weighted by the measured data to control the relative importance of each well log in the inversion procedure. The minimization can be solved using a linearized (Menke, 1984) or a global optimization method (without linearization) such as very fast SA (Sen and Stoffa, 2013) or the float-encoded genetic algorithm (Michalewicz, 1996).

\section{Interval inversion method}

The interval inversion method is based on the establishment of depth-dependent probe response functions. In the forward modeling phase of the inversion procedure, we calculate the $s$ th well log using equation 1 extended to a greater depth interval:

$$
d_{r}^{(\mathrm{cal})}=d^{(\mathrm{cal})}\left(z_{r}\right)=g_{s}\left[m_{1}\left(z_{r}\right), m_{2}\left(z_{r}\right), \ldots, m_{P}\left(z_{r}\right)\right],
$$

where $z_{r}$ is the coordinate of the $r$ th depth $\left(r=1, \ldots, N_{s}\right)$ and $N_{s}$ is the number of the measurement points of the $s$ th well log. The above formulation allows for integrating all the data collected from an arbitrary interval to a joint inversion procedure. To do this, we introduce the integrated data vector as follows:

$$
d^{(\text {cal })}=\left(d_{1}^{1}, \ldots, d_{N_{1}}^{1}, \ldots, d_{1}^{s}, \ldots, d_{N_{s}}^{s}, \ldots, d_{1}^{s}, \ldots, d_{N_{s}}^{s}\right)^{\mathrm{T}},
$$

where $\mathrm{T}$ denotes the symbol of matrix transpose. The $k$ th element of the calculated data vector can be identified as $k=$ $r+N_{1}+N_{2}+\ldots+N_{s-1}$, where index $r$ runs through the data set belonging to the $s$ th well $\log$. The total number of data is $N=N_{1}+N_{2}+\ldots+N_{s}$. The $k$ th calculated data are approximated in a linearized form as follows:

$$
d_{k}^{(\mathrm{cal})}=\sum_{i=1}^{P} G_{k i} m_{i}
$$

In equation 4, the petrophysical parameters are represented as continuous depth functions, whereas the zone parameters neglected from the argument are fixed, but if necessary, they can be treated as depth-dependent quantities, too. We discretize the $i$ th model parameter using a series expansion technique:

$$
m_{i}(z)=\sum_{q=1}^{Q^{(i)}} B_{q}^{(i)} \Psi_{q}(z),
$$

where $B_{q}$ is the $q$ th expansion coefficient, $\Psi_{q}$ is the $q$ th basis function, and $Q^{(i)}$ is the requisite number of expansion coefficients (defined later) in describing the $i$ th model parameter. The basis functions are assumed as known quantities, the selection of which is not strictly limited. In describing a layerwise homogeneous model, we use a combination of unit step functions:

$$
\Psi_{q}(z)=u\left(z-Z_{q-1}\right)-u\left(z-Z_{q}\right)
$$

where $Z_{q-1}$ and $Z_{q}$ are the upper and lower depth coordinates of the $q$ th layer, respectively. Basis function $\Psi_{q}$ in the $q$ th layer equals unity; otherwise it is zero; thus, the $i$ th model parameter in the $q$ th layer can be described by one expansion coefficient, $B_{q}^{(i)}$. The advantage of this formulation is the high overdetermination of the inverse problem. Moreover, the layer-boundary coordinates appearing in equations $6-8$ can be automatically estimated by the interval inversion method (Dobróka and Szabó, 2012).

In this study, we approximate the variation of petrophysical parameters using Legendre polynomials as basis functions:

$$
m_{i}(z)=\sum_{q=1}^{Q^{(i)}} B_{q}^{(i)} P_{q-1}(z)
$$

where the $q$ th degree Legendre polynomial can be written using the Rodrigues formula (McCarthy et al., 1993) as follows:

$$
P_{q}(z)=\frac{1}{2^{q} q !} \frac{d^{q}}{d z^{q}}\left(z^{2}-1\right)^{q} .
$$

The Legendre polynomials form an orthonormal set of functions over the range of -1 and 1 . In the selection of $Q$, one should tend to reduce the number of expansion coefficients to guarantee the numerical stability of the inversion procedure, whereas the proper vertical resolution of petrophysical parameters requires sufficient number of expansion coefficients. By substituting equation 9 to equation 4, the $s$ th well $\log$ can be expressed in terms of the expansion coefficients. The petrophysical parameters no longer constitute the model vector of the inverse problem; instead of them, the expansion coefficients are estimated directly by the inversion procedure. The application of Legendre polynomials is favorable because of the relatively low correlation developing between the model parameters during the inversion process. When we give an estimate to the layer thicknesses by the interval inversion method, the choice of a global optimization algorithm is preferable to avoid numerical problems pertaining to linearization (Dobróka and Szabó, 2011, 2012). According to our tests, assuming given layer thicknesses, the linearized damped least squares (DLSQ) method, suggested by Marquardt (1959), produces a highly stable and fast interval inversion procedure. By combining equations 6 and 7 , we find 


$$
d_{k}^{(\mathrm{cal})}=d^{(\mathrm{cal})}\left(z_{k}\right)=\sum_{i=1}^{P} \sum_{q=1}^{Q^{(i)}} G_{k i} B_{q}^{(i)} \Psi_{q}\left(z_{k}\right) .
$$

Having two indices instead of $B_{q}^{(i)}$, we introduce $B_{l}$ as the $l$ th element of the model vector of the interval inversion problem, where $l=q+Q_{1}+Q_{2}+\ldots+Q_{i-1}$. The total number of unknowns is $M=Q_{1}+Q_{2}+\ldots+Q_{P}$. By replacing the term $G_{k i} \Psi_{q}\left(z_{k}\right)$ with $\tilde{G}_{k l}$, we write the $k$ th calculated data as follows:

$$
d_{k}^{(\mathrm{cal})}=\sum_{l=1}^{M} \tilde{G}_{k l} B_{l}
$$

Depth-coordinate $z_{k}$ and indices $q$ and $i$ belonging to $l$ can be determined by the above reparametrization. The linearized inverse problem can be solved by minimizing the functional given in equation 3 . The $M$-by- 1 vector of expansion coefficients is estimated as follows:

$$
\mathbf{B}=\mathbf{G}^{-g} \mathbf{d}^{(\mathrm{obs})},
$$

where $\mathbf{G}^{-g}$ is the generalized inverse matrix of the DLSQ method (Menke, 1984). The vertical distribution of petrophysical parameters can be derived directly from the interval inversion results using equation 9.

\section{Quality of inversion results}

We check the quality of inversion estimates in the knowledge of the accuracy of input data, which can be provided by repeated welllogging measurements or operation manuals. Horváth (1973) discusses the sources of interpretation errors and gives an estimate to the uncertainty of different types of well-logging data. In the general case, the covariance matrix of the model parameters estimated by a linearized inversion method can be related to the data covariance matrix including the variances of observed data in its main diagonal (Menke, 1984):

$$
\operatorname{cov} \mathbf{m}=G^{-g} \operatorname{cov} \mathbf{d}^{(\mathrm{obs})}\left(\mathbf{G}^{-g}\right)^{\mathrm{T}} .
$$

The dispersion of model parameters is not only the result of data noise, but it is also affected by some amount of modeling errors related to equation 1 . The estimation error of the $i$ th model parameter is derived from equation 14 :

$$
\sigma\left(m_{i}\right)=\left[(\operatorname{cov} \mathbf{m})_{i i}\right]^{1 / 2},
$$

which we use to measure the accuracy of the model parameters estimated by local inversion. One can calculate the covariance matrix of the expansion coefficients $(\operatorname{cov} \mathbf{B})$ estimated by the interval inversion procedure by analogy with equation 14 , where the variance of each datum available in the processing interval is included in the data covariance matrix. The calculation of the accuracy of petrophysical parameters derived from equation 9 requires the propagation of errors taken into consideration. In the knowledge of cov $\mathbf{B}$, we derive the covariance between the petrophysical parameters $m_{i}$ and $m_{j}$ at an arbitrary depth as follows:

$$
[\operatorname{cov} \mathbf{m}(z)]_{i \mathrm{j}}=\sum_{n=1}^{Q^{(i)}} \sum_{m=1}^{Q^{(j)}} \Psi_{n}(z)(\operatorname{cov} \mathbf{B})_{h h^{\prime}} \Psi_{m}(z),
$$

where indices are $h=n+Q_{1}+Q_{2}+\ldots+Q_{i-1}, \quad h^{\prime}=$ $m+Q_{1}+Q_{2}+\ldots+Q_{j-1} \quad(I=1,2, \ldots, P$ and $j=1,2, \ldots, P)$. When applying Legendre polynomials of different degrees as basis functions, equation 9 derives that $\partial m_{i}(z) / \partial B_{q}^{(i)}=P_{q-1}(z)$, which substituted into equation 16 gives

$$
[\operatorname{cov} \mathbf{m}(z)]_{i j}=\sum_{n=1}^{Q^{(i)}} \sum_{m=1}^{Q^{(j)}} P_{n-1}(z)(\operatorname{cov} \mathbf{B})_{h h^{\prime}} P_{m-1}(z) .
$$

Analogously to equation 15 , the estimation error is derived from equation 17, which gives the standard deviation of the $i$ th petrophysical parameter estimated by the interval inversion procedure in the function of logged depth for either correlated or uncorrelated measurements.

The reliability of inversion results can be quantified via the Pearson's correlation matrix (corr $\mathbf{m}$ ). We consider a solution reliable when the model parameters correlate marginally because only uncorrelated (or just poorly correlated) parameters can be resolved uniquely by inversion. If the absolute value of correlation coefficient is close to unity, there is a strong relation between the model parameters referring to unreliable solution. In large-scale inverse problems, it is useful to introduce a scalar for the measure of average correlation:

$$
S(\mathbf{m})=\left\{\frac{1}{P(P-1)} \sum_{i=1}^{P} \sum_{j=1}^{P}\left[(\operatorname{corr} \mathbf{m})_{i j}-\delta_{i j}\right]^{2}\right\}^{1 / 2},
$$

where $\delta$ denotes the Kronecker delta. The above quantity refers to the case of local inversion, which can be extended for checking the reliability of interval inversion results. We define the correlation matrix of expansion coefficients estimated to the entire processing interval as follows:

$$
(\operatorname{corr} \mathbf{B})_{l l^{\prime}}=\frac{(\operatorname{cov} \mathbf{B})_{l l^{\prime}}}{\sigma\left(\mathrm{B}_{l}\right) \sigma\left(\mathrm{B}_{l^{\prime}}\right)},
$$

where indices are $l=1,2, \ldots, M, l^{\prime}=1,2, \ldots, M$ and $M$ is the total number of expansion coefficients estimated by the polynomial-based interval inversion method. The correlation matrix of vector $\mathbf{B}$ can be represented similarly by the scalar defined in equation 18. We quantify the overall misfit between the measured and predicted data of different magnitudes and dimensional units by the relative data distance as follows:

$$
D=\left[\frac{1}{N} \sum_{k=1}^{N}\left(\frac{d_{k}^{(\mathrm{obs})}-d_{k}^{(\mathrm{cal})}}{d_{k}^{(\mathrm{obs})}}\right)^{2}\right]^{1 / 2} \times 100(\%),
$$

where $d_{k}^{(\mathrm{obs})}$ and $d_{k}^{\text {(cal) }}$ are the $k$ th element of the observed and calculated (integrated) data vector, respectively. 


\section{FIELD RESULTS}

\section{Inverse problem}

We test the interval inversion method and compare it to local inversion in a hydrocarbon exploratory borehole (well 1) drilled in the Pannonian Basin in Hungary. In the processed interval, a sedimentary formation of Upper Pannonian (Pliocene) made up of four unconsolidated layers is investigated. Rock samples indicate high-porosity channel sands of good storage capacity interbedded by aleurite laminae and shaly layers. Well-logging data suitable for inversion are represented by natural gamma ray (GR in API), spectral (potassium) gamma ray $(K$ in $\%)$, bulk density $\left(\rho_{\mathrm{b}}\right.$ in $\left.\mathrm{g} / \mathrm{cm}^{3}\right)$, neutron porosity $\left(\Phi_{\mathrm{N}}\right.$ in $\left.\mathrm{v} / \mathrm{v}\right)$, acoustic traveltime $(\Delta t$ in $\mu \mathrm{s} / \mathrm{ft})$, and deep laterolog resistivity $\left(R_{\mathrm{d}}\right.$ in ohm-m) logs. The depth-matched and environmentally corrected well logs transformed into the depth interval of $0-19.3 \mathrm{~m}$ are plotted in Figure 1. In the last track, the combination of density and neutron logs indicates the presence of hydrocarbons in the second and fourth layers. The pore fluid was identified previously as methane, ethane, propane, and a small amount of carbon dioxide. In the lack of observed information on data variances, we assume that the well logs are of different uncertainties. We give the data covariance matrix in equation 14 :

$$
\operatorname{cov} \mathbf{d}^{(\mathrm{obs})}=\operatorname{diag}\left(\sigma_{\mathrm{GR}}^{2}, \sigma_{K}^{2}, \sigma_{\rho_{\mathrm{b}}}^{2}, \sigma_{\Phi_{N}}^{2}, \sigma_{\Delta t}^{2}, \sigma_{R_{\mathrm{d}}}^{2}\right) .
$$

For studying the effect of data variance on the solution of the inverse problem, we estimate the standard deviations of data, similarly to Horváth (1973), as $\sigma_{\mathrm{GR}}=0.08, \sigma_{K}=0.07, \sigma_{\rho_{\mathrm{b}}}=0.05$, $\sigma_{\Phi_{\mathrm{N}}}=0.09, \sigma_{\Delta t}=0.06$, and $\sigma_{R_{\mathrm{d}}}=0.06$. The confidence intervals of well logs measured in well 1 are indicated in Figure 1. lows:

The general form of the $k$ th probe response equation is as fol-

$$
d_{k}=f\left(\Phi, S_{\mathrm{x} 0}, S_{\mathrm{w}}, V_{\mathrm{sh}}, V_{\mathrm{sd}}, c\right)
$$

where effective porosity $\Phi(\mathrm{v} / \mathrm{v})$, shale volume $V_{\mathrm{sh}}(\mathrm{v} / \mathrm{v})$, sand volume $V_{\mathrm{sd}}(\mathrm{v} / \mathrm{v})$, water saturation in the invaded $S_{\mathrm{x} 0}(\mathrm{v} / \mathrm{v})$, and that of uninvaded zone $S_{\mathrm{w}}(\mathrm{v} / \mathrm{v})$ are the unknowns of the inverse problem. To simulate nuclear logs (GR, $K, \rho_{\mathrm{b}}$, and $\Phi_{\mathrm{N}}$ ), linear response functions corrected for shale, mudfiltrate, and hydrocarbon effects are used suggested for shaly sand interpretation by Baker Atlas (1996). For calculating the sonic response $(\Delta t)$, the compaction corrected time-average formula is applied (Wyllie et al., 1956). The deep resistivity $\left(R_{\mathrm{d}}\right) \log$ is calculated first by different nonlinear models, that is, the Archie formula (Archie, 1942), Simandoux equation (Simandoux, 1963), total shale relationship (Schlumberger, 1989), and Indonesia model (Poupon and Leveaux, 1971). By solving the interval inversion problems separately, the overall data distance based on equation 20 (calculated only for resistivity data) is $2.8 \%$ for the Archie equation, $3.4 \%$ for the Indonesia model, $2.6 \%$ for the Schlumberger model, and $4.4 \%$ for the Simandoux model. Because it gives the lowest misfit, we choose the total shale model for testing the local and interval inversion procedure. In equation 22, the zone parameters in vector $\mathbf{c}$ representing the physical properties of mud filtrate, hydrocarbon, shale, and sand are treated as constant in the local inverse problem (Table 1). They can be estimated from the literature, drilling information, core measurements, crossplot techniques, trial-and-error methods, or some of them alternatively by interval inversion (Dobróka and Szabó, 2011). The material balance equation used to constrain the estimation of petrophysical parameters is $\Phi+V_{\mathrm{sh}}+V_{\mathrm{sd}}=1$. Equation 22 does not contain the layer-boundary coordinates, which cannot be extracted by local inversion methods. They can be a priori given from manual analysis of the GR or shallow resistivity logs, determined by multivariate statistical methods, such as cluster analysis, fuzzy logic, and neural networks (Maiti et al., 2007), or they can be estimated automatically by interval inversion (Dobróka and Szabó, 2012).

In solving the inverse problem, we apply two different approaches. The traditional is represented by the local inversion method, which extracts the petrophysical parameters depth by depth with the subsequent analysis of local data sets. On the contrary, the interval inversion method integrates all the data collected from the logged interval to produce the well logs of petrophysical parameters in a joint inversion procedure. The discretization of model parameters is made by equation 9, which can be applied to an arbitrary depth interval. We suggest specifying the depth interval of series expansion automatically by using a more sophisticated interval inversion algorithm. In the first phase, an interval inversion problem is solved using equation 8 to give an estimate to layer thicknesses and petrophysical parameters of a homogeneous layer model using the SA method. We choose equation 3 as an energy function extended to the entire processing interval. The following cooling schedule assures to achieve the global optimum (Geman and Geman, 1984):

$$
T(\nu)=\frac{T_{0}}{\ln (\nu)},
$$

where $T_{0}$ denotes an appropriate initial temperature and $\nu$ is the number of iteration steps. At the end of the SA procedure, we separate different layers in which the estimated constants of petrophys-

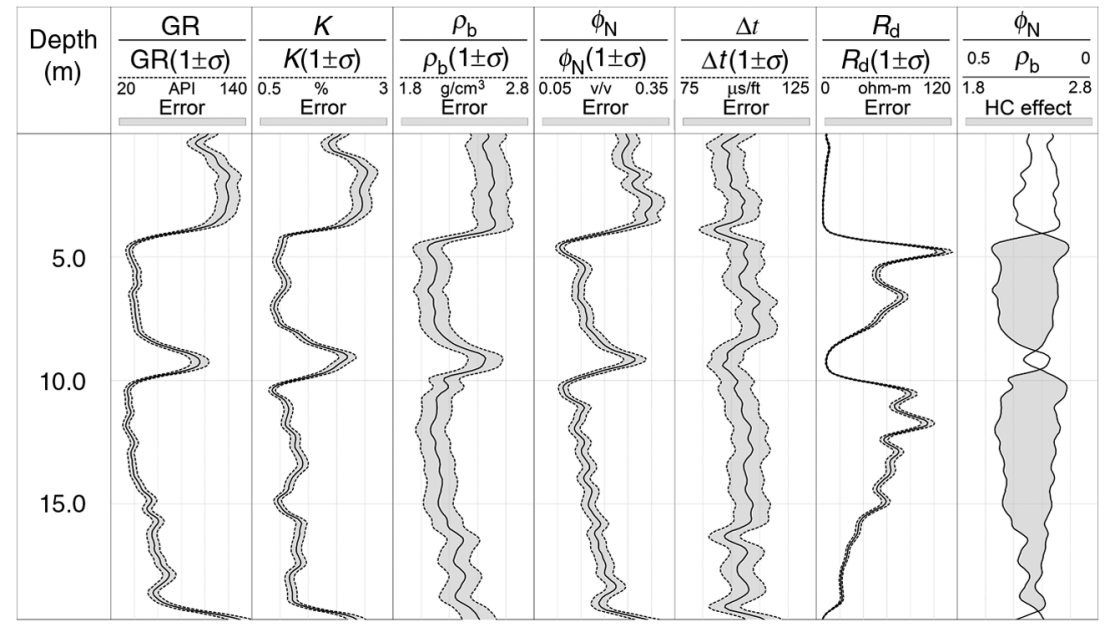

Figure 1. Input well logs measured in well 1 and uncertainty (error) ranges of log readings for local and interval inversion procedures: GR is natural gamma ray, $K$ is potassium, $\rho_{\mathrm{b}}$ is bulk density, $\Phi_{\mathrm{N}}$ is neutron porosity, $\Delta_{t}$ is acoustic traveltime, $R_{\mathrm{d}}$ is resistivity, and $\sigma$ is standard deviation of observed data types. 
ical parameters can be considered as initial values of expansion coefficients of Legendre polynomials of the zeroth order in equation 9. In the second phase, we refine the vertical distributions of petrophysical parameters within the separated intervals in distinct interval inversion procedures using the Legendre polynomials as basis functions. For this purpose, we perform a DLSQ-based interval inversion of well logs to give a fast and stable solution.

In the inversion procedures analyzed in this study, we use the same forward problem solution. We show that the set of response equations applied in this study gives a proper fit between measured and calculated data in well 1 . In case of more complex geologic situations, the forward modeling equations can be further developed, for example, by Drahos (1984), Tang and Cheng (1993), and Mendoza et al. (2010), which can be easily implemented in the interval inversion procedure. In this study, we emphasize the improvement of the inversion algorithm. Our aim is to develop a powerful inversion tool that provides better estimation accuracy than local inversion procedures. This is attained by the significant increase of overdetermination using a Legendre polynomial-based discretization strategy. Therewith, the treatment of zone parameters as inversion unknown can be considered as a step forward in the forward problem solution, too. In our conception, it is a petrophysics-based innovation that we give an objective estimate to zone parameters instead of fixing them arbitrarily during the inversion procedure.

\section{Comparative study}

We compare the polynomial series expansion-based interval inversion method to the local inversion procedure. The aim of the present task is to determine the vertical distributions of quantities $\Phi, S_{\mathrm{x} 0}, S_{\mathrm{w}}, V_{\mathrm{sh}}, V_{\mathrm{sd}}$ in well 1 . Because we have six types of data in each depth (GR, $K, \rho_{\mathrm{b}}, \Phi_{\mathrm{N}}, \Delta t$, and $R_{\mathrm{d}}$ ) and we can estimate the sand volume deterministically from the material balance equation, the overdetermination ratio in case of depth-by-depth inversion is 1.5. (We have four unknowns in each depth, totally 776 unknowns in the investigated interval.) In local inversion, we first set an initial model by giving a first guess to the model parameters. In permeable layers, we assume the starting values of $\Phi=S_{\mathrm{w}}=V_{\mathrm{sh}}=0.2 \mathrm{v} / \mathrm{v}$, $S_{\mathrm{x} 0}=0.7 \mathrm{v} / \mathrm{v}$, whereas in impermeable beds, we set them as $\Phi=0.1 \mathrm{v} / \mathrm{v}, S_{\mathrm{x} 0}=S_{\mathrm{w}}=1.0 \mathrm{v} / \mathrm{v}, V_{\mathrm{sh}}=0.6 \mathrm{v} / \mathrm{v}$. We do not allow the water saturation to exceed unity. We use the DLSQ algorithm to solve the local inverse problem. Because the condition number of matrix $\mathbf{G}^{\mathrm{T}} \mathbf{G}$ derived from equation 2 is occasionally $2 \times 10^{3}-1.2 \times 10^{4}$, we choose the regularization factor $\varepsilon^{2}$ in equation 3 as 15 , which we decrease progressively down to $3 \times$ $10^{-5}$ in 10 iteration steps. We calculate the data distance defined in equation 20 to each depth the average of which is $3.7 \%$ at the end of the inversion procedure. The CPU time of the inversion process is $11 \mathrm{~s}$ using a quad-core processor workstation. The local inversion results are illustrated in Figure 2. Tracks 1-6 show a close fit between the observed and calculated data. The well logs of the estimated petrophysical parameters are in tracks 7-8. In the saturation track, the movable and irreducible hydrocarbon saturations are derived from the inversion results by $S_{\mathrm{hm}}=S_{\mathrm{x} 0}-$ $S_{\mathrm{w}}$ and $S_{\text {hir }}=1-S_{\mathrm{x} 0}$, respectively. The fractions of porosity $(\Phi=0.04-0.28 \mathrm{v} / \mathrm{v})$, shale $\left(V_{\mathrm{sh}}=\right.$ $0.08-0.71 \mathrm{v} / \mathrm{v})$, sand $\left(V_{\text {sd }}=0.21-0.69 \mathrm{v} / \mathrm{v}\right)$ relative to the unit volume of rock are plotted in track 8 .

In the next step, we estimate the distribution of petrophysical parameters in the entire logging interval by the DLSQ-based interval inversion procedure. By a sampling distance of $0.1 \mathrm{~m}$, we have totally 1164 data. We discretize the model parameters $\left(\Phi, S_{\mathrm{x} 0}, S_{\mathrm{w}}, V_{\mathrm{sh}}\right)$ using equation 9 with applying a set of Legendre polynomials of up to 44 degrees as basis functions. (The optimal choice of polynomial degree will be discussed two subsections later.) The total number of unknowns is $4(Q *+1)=180(Q *$ is the maximum degree of the Legendre polynomials); thus, the data-to-unknowns ratio is 6.5 . The relative increase in overdetermination ratio is more than $330 \%$ compared with local inversion. We 
choose the initial values of expansion coefficients as $B_{1}=0.1$ for $\Phi, B_{1}=0.5$ for $S_{\mathrm{x} 0}, B_{1}=0.2$ for $S_{\mathrm{w}}$ and $V_{\text {sh }}$, respectively, whereas $B_{q}=0(q=2,3, \ldots, 45)$ is applied to every petrophysical parameter. We calculate the $1164 \times 180$ Jacobi's matrix in each iteration step, whereas the condition number of the $180 \times 180$ matrix $\mathbf{G}^{\mathrm{T}} \mathbf{G}$ is approximately 300 . Thus, we do not have to apply the regularization term in equation 3 and the inversion procedure is still stable at $\varepsilon^{2}=0$. We set the maximum number of iteration steps to 10 . The data distance is $728 \%$ in the first step, which decreases down to $4.4 \%$ after six iteration steps. The CPU time of the inversion process is $1 \mathrm{~min} 56 \mathrm{~s}$ using the same workstation.

The relative increase of processing time is 9.5 compared with local inversion. The expansion coefficients with their estimation errors calculated by equation 16 are illustrated in Figure 3. The relative errors of coefficient $B_{1}$ is $3 \%-6 \%$. The standard deviation of porosity and shale content is smaller than that of the water saturation. The $180 \times 180$ correlation matrix of expansion coefficients calculated by equation 19 is illustrated in Figure $4 c$, whereas the $4 \times 4$ correlation matrices of model parameters estimated by local inversion in an impermeable layer $(z=2 \mathrm{~m})$ and in a permeable bed $(z=12 \mathrm{~m})$ are plotted in Figure $4 \mathrm{a}$ and $4 \mathrm{~b}$, successively. The correlation average defined in equation 18 shows that the strength of correlation between the model parameters is much smaller for interval inversion; that is, the relative decrease in the average of absolute values of correlation coefficients is $70 \%$, which refers to a more reliable estimation for the inversion unknowns. The small value of correlation average explains the great stability of the interval inversion procedure. The result of well log analysis is illustrated in Figure 5. The calculated well logs are in close agreement with the observed ones (tracks 16). The variation of water saturation along the borehole is somewhat smoother compared with the case of local inversion (track 7). The relative volumes of porosity $(\Phi=0.03-0.28 \mathrm{v} / \mathrm{v})$, shale $\left(V_{\mathrm{sh}}=0.07-0.71 \mathrm{v} / \mathrm{v}\right)$, and sand $\left(V_{\mathrm{sd}}=\right.$ $0.24-0.69 \mathrm{v} / \mathrm{v})$ are plotted in track 8 .

The estimation errors of petrophysical parameters can be compared in Figure 6, which are calculated by equation 15 . The standard deviation of water saturation is one order of magnitude higher than that of the porosity and shale volume. In permeable layers, the estimation errors are relatively small, but in impermeable beds, they abruptly increase because of the stronger correlation between the model parameters. In tracks 6-7, the high values of correlation averages with small data distances indicate an ambiguous interpretation of water saturation in shaly intervals, which makes the estimation error of petrophysical parameters locally increase. The overall result shows that the interval inversion procedure improves the estimation accuracy compared with the local inversion method. We achieve $60 \%$ rel- ative decrease in the average standard deviation of porosity, $52 \%$ in that of water saturation in the uninvaded zone, $55 \%$ in that of shale content, $50 \%$ in that of water saturation in the invaded zone. The improvement is well marked in impermeable intervals, whereas the use of the interval inversion method is the most favorable in hydrocarbon reservoirs, where the absolute value of estimation error is the smallest. The improvement of estimation accuracy for porosity and water saturations in the fourth layer is demonstrated in Figure 7. The correlation coefficients for petrophysical parameters estimated by local inversion can be compared with those of the interval inversion

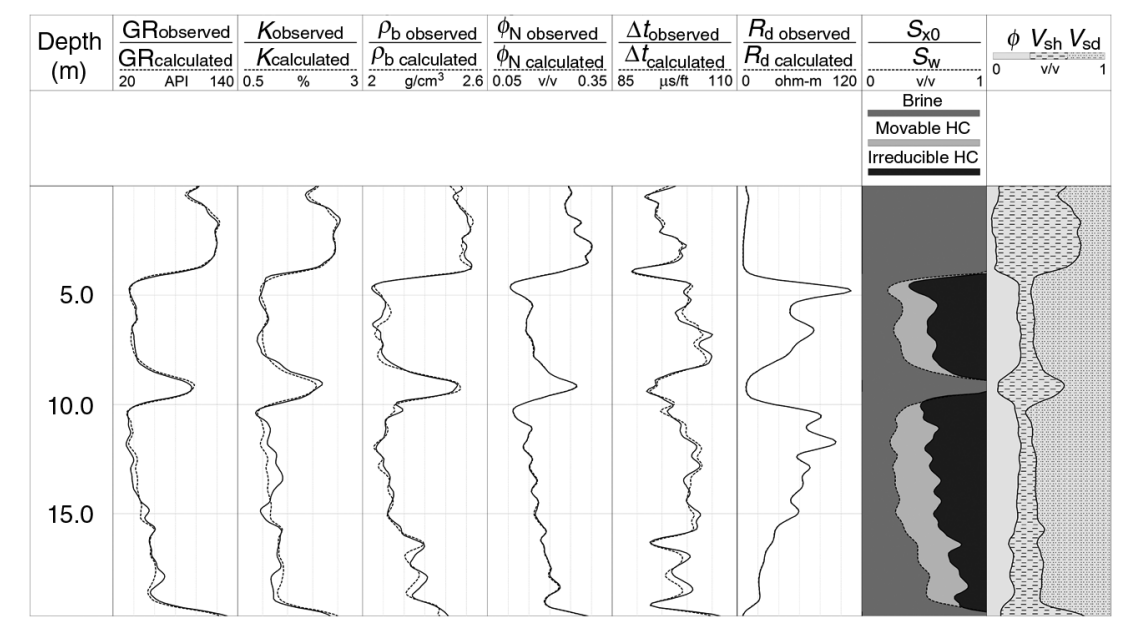

Figure 2. Local inversion results in well 1. Observed (solid line) and calculated (dashed line) well logs: GR is natural gamma ray, $K$ is potassium, $\rho_{\mathrm{b}}$ is bulk density, $\Phi_{\mathrm{N}}$ is neutron porosity, $\Delta t$ is acoustic traveltime, $R_{\mathrm{d}}$ is resistivity. Petrophysical parameters estimated by local inversion are: $\Phi$ is effective porosity, $S_{\mathrm{x} 0}$ is water saturation (invaded zone), $S_{\mathrm{w}}$ is water saturation (uninvaded zone), and $V_{\mathrm{sh}}$ is shale volume. Derived parameters are sand volume $V_{\text {sd }}$, brine saturation, and movable and irreducible hydrocarbon (HC) saturation.
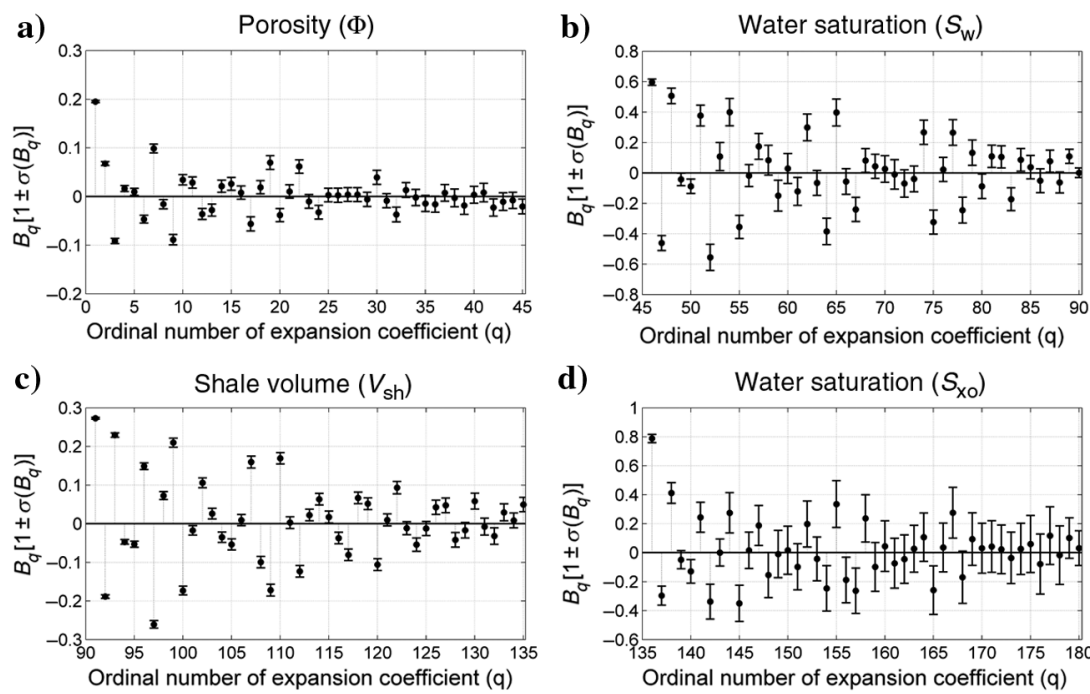

Figure 3. Results of interval inversion procedure using Legendre polynomials of 44 degrees as basis functions in well 1. Estimated values of expansion coefficients for (a) porosity, (b) water saturation of uninvaded zone, (c) shale content, (d) water saturation of invaded zone, and their estimation error ranges versus ordinal number of expansion coefficients in the model vector. 
results in Figure 8. The overall strength of correlation between the volumetric parameters is weaker in case of interval inversion. The improvement is more significant in shaly layers than in the hydro-
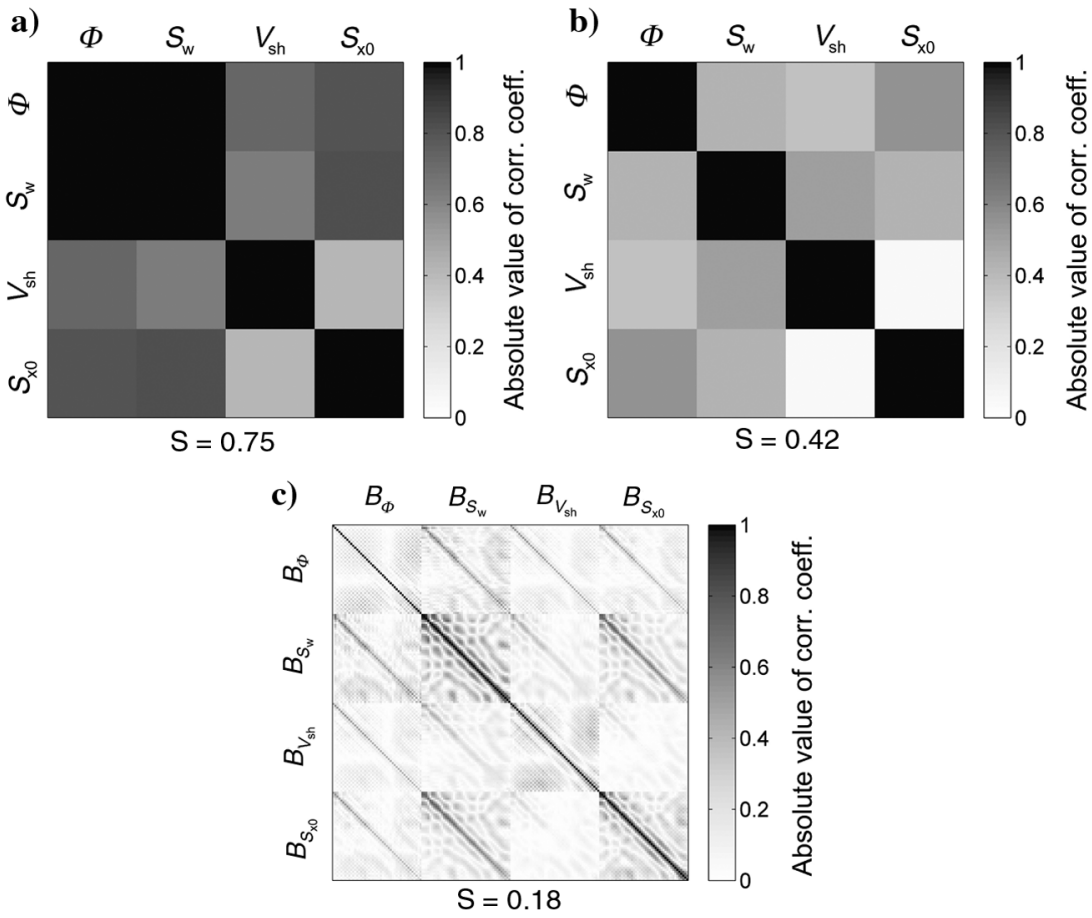

Figure 4. Correlation matrices of inversion estimates in well 1. Absolute values of Pearson's correlation coefficients estimated by local inversion in (a) depth $2 \mathrm{~m}$ and (b) depth $12 \mathrm{~m}$, estimated by (c) interval inversion in 0-19.3 m. The petrophysical parameters are as follows: $\Phi$ is effective porosity, $S_{\mathrm{x} 0}$ is water saturation (invaded zone), $S_{\mathrm{w}}$ is water saturation (uninvaded zone), and $V_{\mathrm{sh}}$ is shale volume. $S$ is the average correlation between the model parameters; $\mathbf{B}$ is the vector of expansion coefficients.

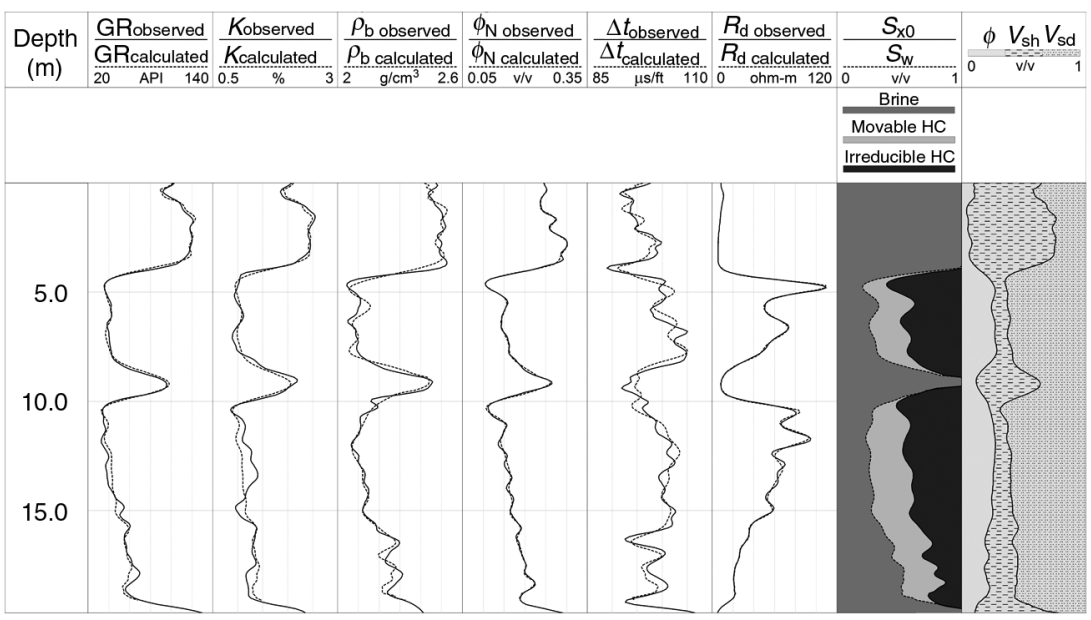

Figure 5. Interval inversion results in well 1. Observed (solid line) and calculated (dashed line) well logs: GR is natural gamma ray, $K$ is potassium, $\rho_{\mathrm{b}}$ is bulk density, $\Phi_{\mathrm{N}}$ is neutron porosity, $\Delta t$ is acoustic traveltime, $R_{\mathrm{d}}$ is resistivity. Petrophysical parameters estimated by interval inversion are: $\Phi$ is effective porosity, $S_{\mathrm{x} 0}$ is water saturation (invaded zone), $S_{\mathrm{w}}$ is water saturation (uninvaded zone), and $V_{\mathrm{sh}}$ is shale volume. Derived parameters are: $V_{\text {sd }}$ is sand volume, brine saturation, and movable and irreducible hydrocarbon (HC) saturation. carbon reservoirs. The average correlation between the petrophysical parameters is 0.44 for interval inversion, which means a relative decrease of $12 \%$ as opposed to local inversion. Almost an entire correlation between porosity and water saturation causes the large estimation errors in the first and third layers. The water saturations of the invaded and virgin zone are also strongly correlated to each other. In these cases, one of them should be estimated out of the inversion procedure. Figure 9 demonstrates a good correlation between the porosities estimated independently by the interval inversion procedure, CLASS deterministic approach (Baker Atlas, 1996) and core data available from neighboring boreholes (well 2 is located approximately $1300 \mathrm{~m}$, whereas well 3 is approximately $500 \mathrm{~m}$ away from well 1). The hydrocarbon formation correlate well between the boreholes and the interval inversion results show a close fit to those of the independent evaluation procedures. As a conclusion, the interval inversion method gives more accurate and reliable estimation in a more stable inversion procedure compared with local inversion. In the presented case, the interval inversion method gives a smoother but more accurate solution with slightly worse data misfit. The vertical resolution depends on the selection of the number of unknowns of the inverse problem.

\section{Determination of interval length}

The major increase of the number of expansion coefficients in the same depth interval can improve the vertical resolution of the interval inversion method, but it may also lead to the largescale decrease of overdetermination and estimation accuracy. One can also enlarge the resolution capability of the interval inversion method by separating the processed length into smaller intervals in which one can expand the petrophysical parameters into series with sufficient number of expansion coefficients.

We execute the SA-based interval inversion procedure using the discretization scheme 8 as a preliminary data processing step to separate different lithologic units and designate their boundaries in well 1 . We repeat the test 20 times to verify the solution. We apply the cooling schedule given in equation 23 with $T_{0}$ of 0.01-0.07 and a maximal number of iteration steps $\nu_{\max }=6000$ in each inversion run. The maximum value of perturbation is $0.1 \mathrm{v} / \mathrm{v}$ and $3 \mathrm{~m}$ for the petrophysical parameters and layer thicknesses, respectively, which is decreased down by $80 \%$ in each 500th iteration step. The average of initial data distances (i.e., energies) is $50 \%$, which is progressively decreased down to $7.6 \%$ at the end of the inversion procedure. This value is because of the layerwise homogeneous model approximation, which will 
be improved by the Legendre polynomial-based inversion phase. The steady convergence of the SA procedure is shown in Figure 10, in which the average energy calculated in each iteration step with its standard deviation is illustrated. The optimal values of layer boundaries are found after a couple of hundred steps (the average is 500), and then only the petrophysical parameters are refined continuously. The results of the SA procedure are listed in Table 2, which specifies four intervals (layer boundaries) and provides the average values and standard deviations of petrophysical parameters as initial values of series expansion coefficients for the subsequent inversion phase.

The input well logs and the resultant layer thicknesses given by the SA procedure are plotted in Figure 11. We find the layer boundary coordinates in depths 4.2, 8.5, 9.9, and $19.2 \mathrm{~m}$. Afterward, we solve a set of interval inversion problems in four separated beds, respectively. We use a different number of expansion coefficients layer by layer, whereas $Q$ in equation 9 is the same for every petrophysical parameters. We choose the degree of Legendre polynomials as $18,18,8$, and 24 in the subsequent beds (from the top). We initialize the values of coefficient $B_{1}$ of each model parameter from the estimates of the SA procedure (Table 2). The overdetermination ratios are 3.2, 3.9, 1.7, and 3.5 in the layers, separately. The maximum number of iterations is 10 in each DLSQ inversion procedure (Figure 10). After completing the inversion procedures, we obtain the optimal data misfits in the four layers as $1.7 \%, 3.6 \%, 3.0 \%$, and $5.7 \%$, respectively. The average of data distances is $3.5 \%$, whereas the overall estimation error of petrophysical parameters shows $10 \%$ relative decrease in the four layers. The well logs of the petrophysical parameters are plotted in Figure 11. The overdetermination ratio is only a double than in local inversion. However, the average correlations for the expansion coefficients are 0.20, $0.19,0.30$, and 0.44 , respectively, and the average of them $(S=0.28)$ shows a $52 \%$ relative improvement demonstrating the stability of the series expansion-based interval inversion procedure. It is concluded that the accuracy of estimation is dependent on the degree of overdetermination. A trade-off must be taken between the vertical resolution and estimation accuracy, which should be based on the proper selection of the number of expansion coefficients.

\section{Selection of the optimal number of expansion coefficients}

We suggest a technique for choosing the optimal number of expansion coefficients for the interval inversion procedure applied to an a)

b)

c)

d)

e)

f)

g

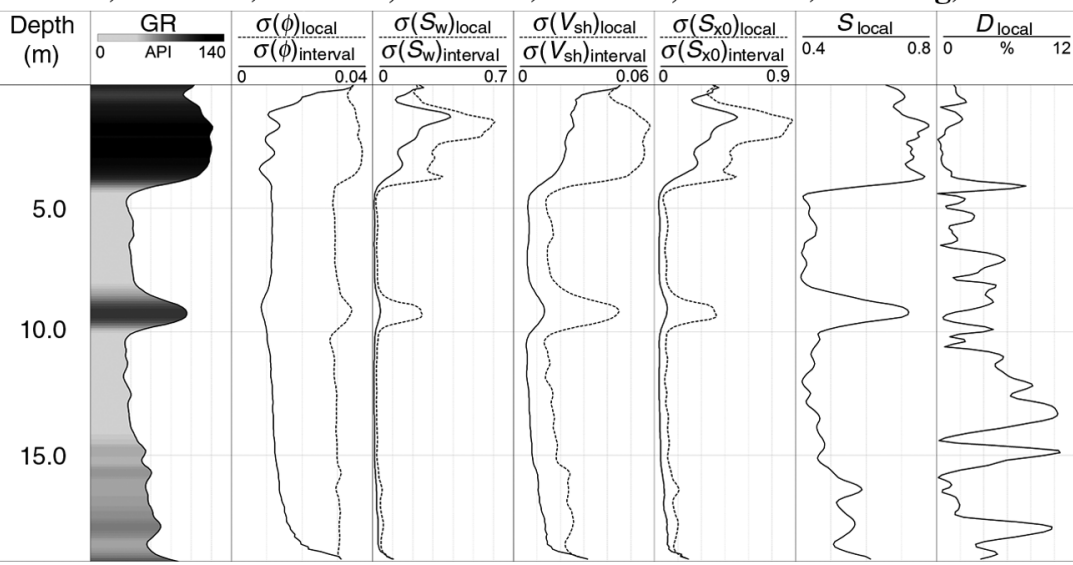

Figure 6. Well logs of standard deviations, $\sigma$, estimated by local and interval inversion methods in well 1 (b-e). Auxiliary well logs are as follows: GR is natural gamma ray (a), $S$ is correlation average of model parameters estimated by local inversion (f), and $D$ is the relative data distance estimated by local inversion $(\mathrm{g})$.

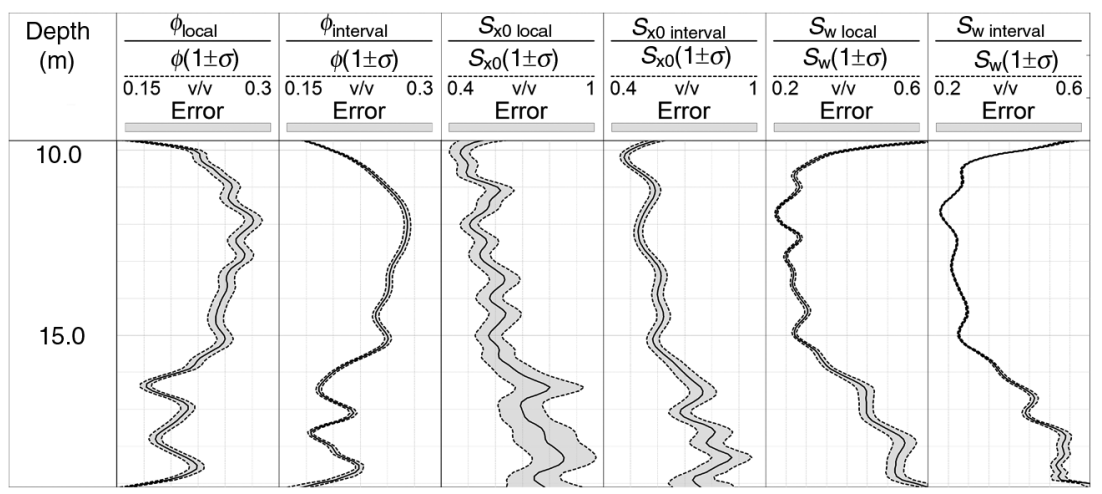

Figure 7. Well logs of petrophysical parameters estimated by the local and interval inversion procedure, separately. Solid lines represent the estimated values of effective porosity $\Phi$, water saturation for the invaded zone $S_{\mathrm{x} 0}$ and for the uninvaded zone $S_{\mathrm{w}}$. Dashed lines show the error bounds of petrophysical parameters calculated from the standard deviations $\sigma$ of inversion estimates.

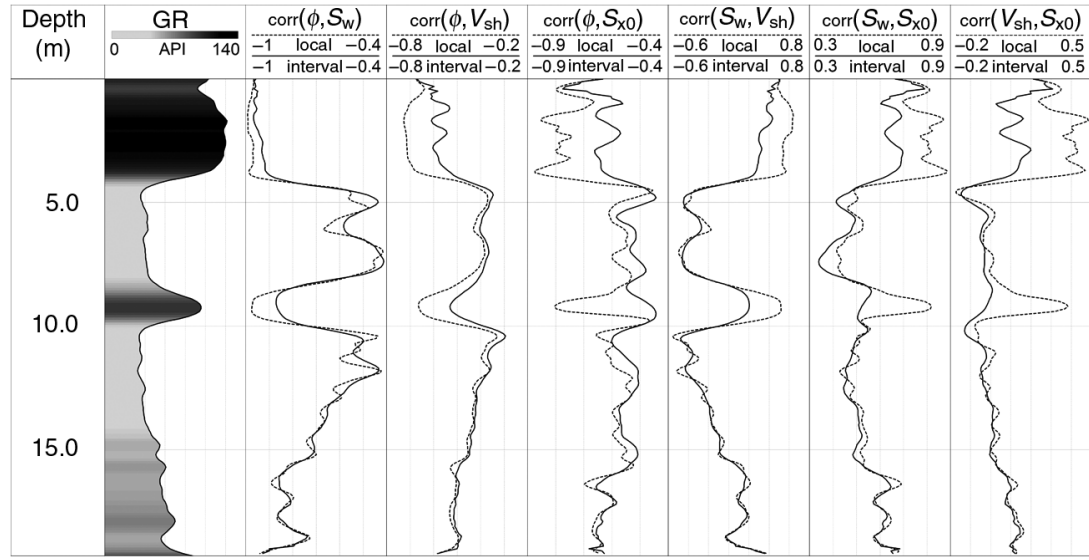

Figure 8. Well logs of correlation coefficients estimated by local and interval inversion methods in well 1. GR as natural gamma ray (left panel) log is plotted for reference. 
arbitrarily chosen depth interval. As an example, we evaluate the hydrocarbon reservoir situated between 9.7 and $19.2 \mathrm{~m}$ in well 1. We perform a set of interval inversion procedures in the same layer by different number of expansion coefficients using equation 9. We summarize the test results in Figure 12. In the beginning, we start with one expansion coefficient per petrophysical parameter. This choice could work well in homogeneous beds, but in this inhomogeneous one, it gives only a rough approximation. Despite the overdetermination ratio (higher than 142), the misfit between the measured and predicted data is extremely high $(D=25 \%)$. The strength of the correlation between the expansion coefficients is moderate $(S=0.47)$. By increasing the number of expansion coefficients, we make a continuous decrease in the data distance and average correlation. In the optimum (25 unknowns per petrophysical parameter), the overdetermination ratio is high enough (almost $6.0)$ to give reliable $(S=0.11)$ inversion estimates by an accepted value of data misfit $(D=5.9 \%)$. (This prediction error is even smaller in surrounding beds.) A further increase in the number of expansion coefficients implies the decrease of overdetermination and negligible improvement in data distance. For instance, the use of 41 expansion coefficients per petrophysical parameter results in higher correlation $(S=0.44)$ again and one order of magnitude higher average of relative estimation errors compared with the optimal case. Such an overparametrized inversion procedure is also time consuming, which does not give much more detailed or accurate information on the petrophysical properties of formations. We suggest to perform preliminary program test runs to set the optimal values of control parameters for a more accurate and reliable inverse modeling.
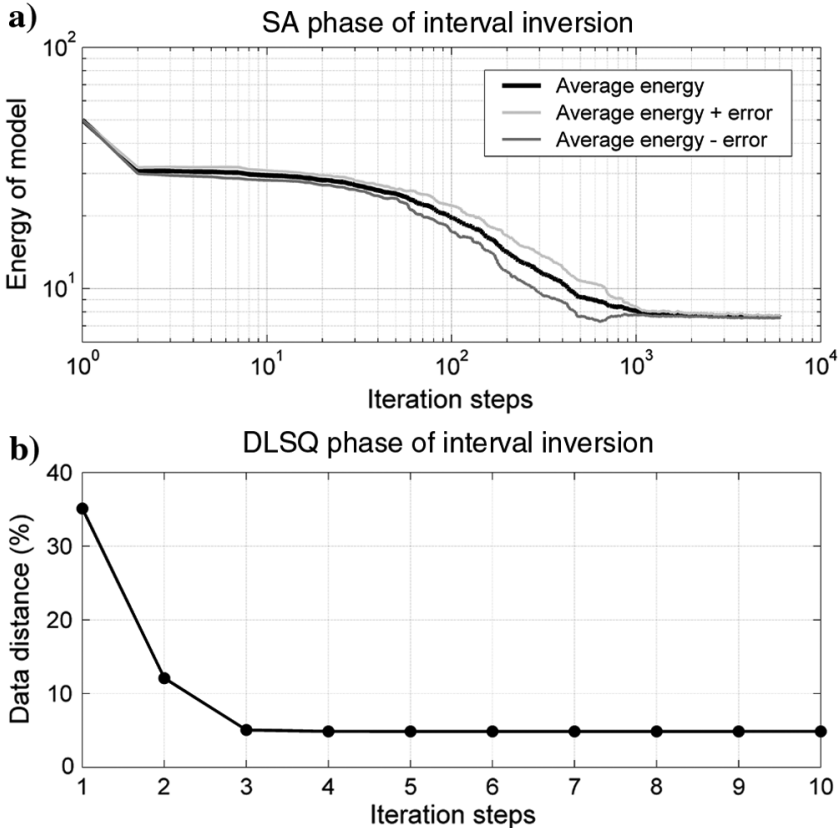

Figure 10. Convergence plots of the global and linear interval inversion procedures. (a) Trend of energy versus number of iterations after 20 independent SA inversion runs using unit-step basis functions. Average energy calculated in each iteration step (black line), average energy \pm standard deviation of energies (light- and darkgray curves). (b) Development of convergence in the subsequent DLSQ inversion procedure using Legendre polynomials as basis functions.
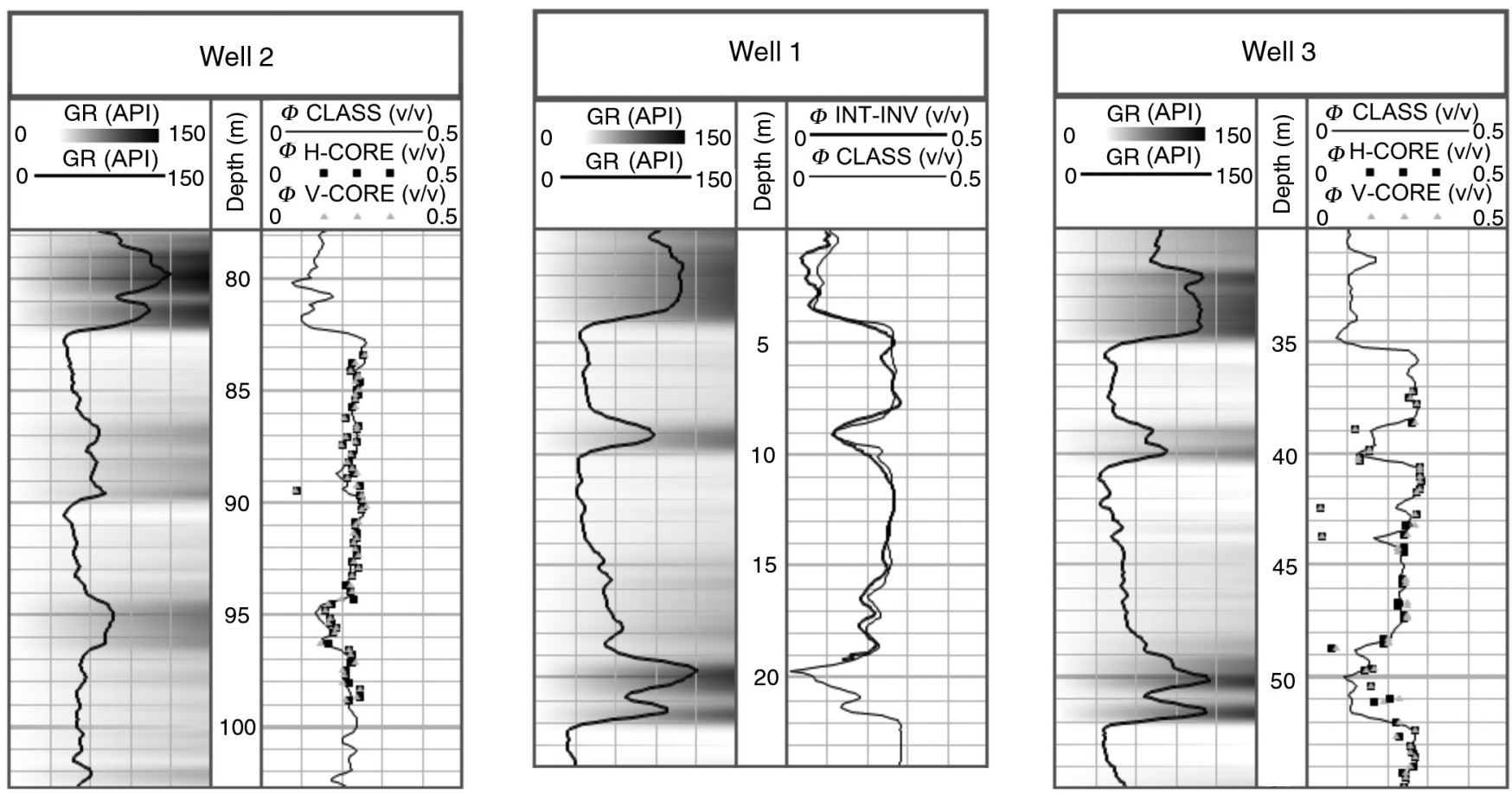

Figure 9. Porosity estimated by interval inversion procedure, deterministic modeling, and core measurements in wells $1-3$. Well logs are as follows: GR is natural gamma ray, $\Phi$ is effective porosity estimated from interval inversion (INT-INV), CLASS interpretation system (CLASS), horizontal (H-CORE), and vertical (V-CORE) core data. 


\section{Estimation of zone parameters}

In the local inversion methodology, zone parameters appearing in equation 22 (detailed in Table 1) are usually fixed to avoid the solution of an ambiguous underdetermined inverse problem. The greatly overdetermined interval inversion procedure allows for the automated determination of zone parameters without a major decrease in the data-to-unknowns ratio. By estimating the zone parameters within the inversion procedure, we improve the solution of the inverse problem and the forward problem implicitly. Previously, we introduced the relevant parameter sensitivity functions, defined as the extent of influence on well-logging data exerted by textural parameters, and we showed that only highly sensitive zone parameters may be applicable as unknown for the interval inversion problem (Dobróka and Szabó, 2011). Those can be determined uniquely by inversion, which correlates weakly to other petrophysical or zone parameters. The advantage of the interval inversion method is that it provides an objective estimate to zone parameters with their estimation errors to the processed interval(s).

Based on the parameter-sensitivity tests, we choose suitable zone parameters to be estimated in the DLSQ-based interval inversion procedure. In the first step, the petrophysical parameters $\left(\Phi, S_{\mathrm{x} 0}\right.$, $S_{\mathrm{w}}$, and $V_{\mathrm{sh}}$ ) are discretized using Legendre polynomials of $44 \mathrm{de}-$ grees as basis functions in well 1 . One zone parameter of the zerothorder approximation is added to each inversion procedure, which we call separate inversion in Table 3. In these cases, the total number of unknowns is $4(Q *+1)+1=181$ and the overdetermination ratio is 6.4 in the processing interval of $19.3 \mathrm{~m}$. The relative decrease of the data-tounknowns ratio compared with the interval inversion with fixed zone parameters is $1.1 \%$, whereas the relative increase of that is $329 \%$ compared with local inversion. In the separate inversion procedures, we can fix parameter $\varepsilon^{2}$ as zero, and the maximum number of iteration steps is 10. The inversion results in Table 3 confirm that we provide a proper estimate to shale, matrix, and fluid properties in stable inversion procedures. The estimation errors of the zone param-

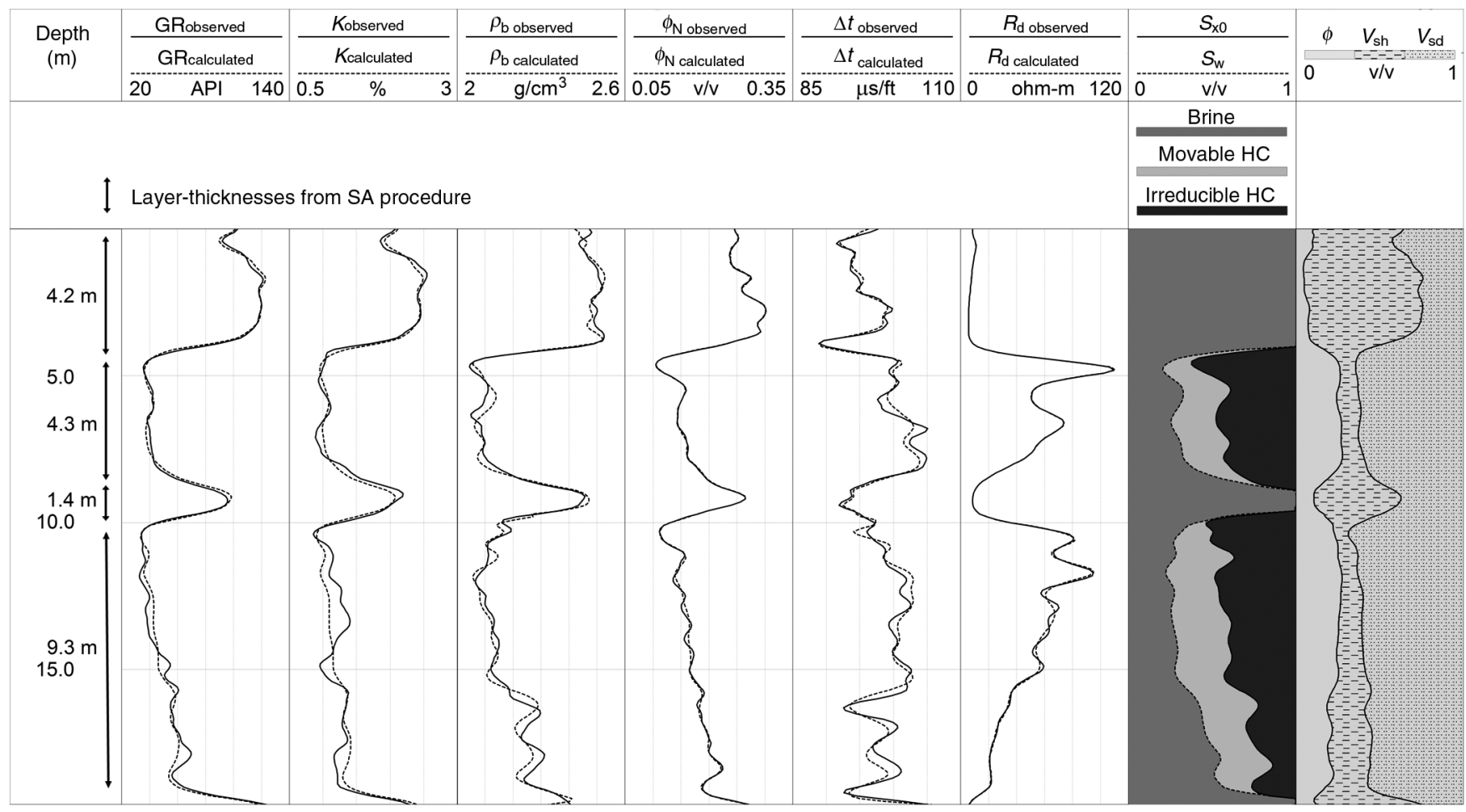

Figure 11. Legendre polynomial-based interval inversion results in well 1. Layer thicknesses are estimated by SA-based layer-by-layer interval inversion are indicated in the depth scale. Observed (solid line) and calculated (dashed line) well logs are as follows: GR is natural gamma ray, $K$ is potassium, $\rho_{\mathrm{b}}$ is bulk density, $\Phi_{\mathrm{N}}$ is neutron porosity, $\Delta t$ is acoustic traveltime, and $R_{\mathrm{d}}$ is resistivity. Petrophysical parameters estimated by interval inversion are effective porosity $\Phi$, water saturation (invaded zone) $S_{\mathrm{x} 0}$, water saturation (uninvaded zone) $S_{\mathrm{w}}$, and shale volume $V_{\mathrm{sh}}$. Derived parameters are sand volume $V_{\mathrm{sd}}$, brine saturation, and movable and irreducible hydrocarbon (HC) saturation. 
eters are proportional to data variances. In the data distance, a slight improvement is detected. The zone parameters correlate to each other and the petrophysical parameters weakly. The average correlation of inversion unknowns is $S=0.18$. Parameter $S_{1}$ in Table 3 represents the average of absolute value of correlation coefficients between the given zone parameter and all the other expansion co-

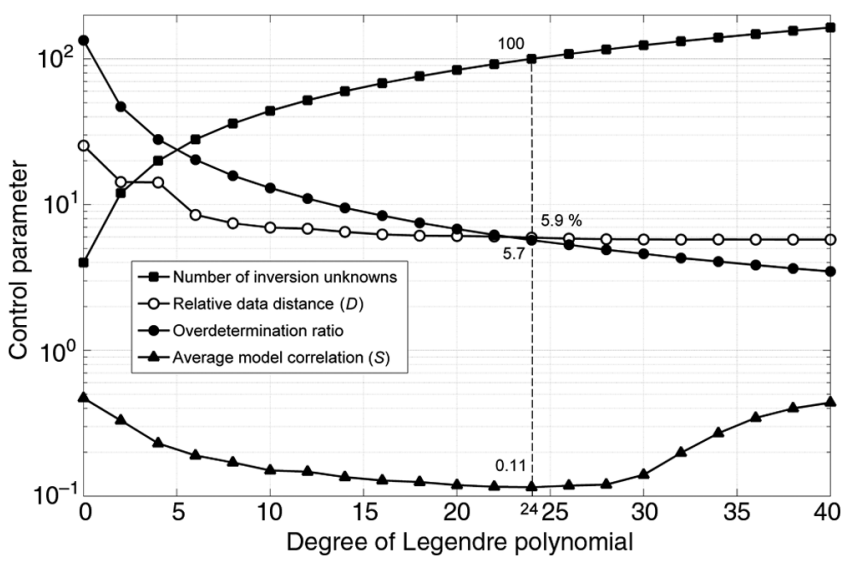

Figure 12. Interval inversion test results in an inhomogeneous gasbearing layer $(9.7-19.2 \mathrm{~m})$ in well 1 . Total number of expansion coefficients estimated by inversion (box symbol), relative data misfit (circle symbol), data-to-unknowns ratio (full-circle symbol), average of correlation coefficients between expansion coefficients (triangle symbol) versus degree of Legendre polynomials applied as basis functions.

Table 3. Zone parameters estimated by interval inversion of well logs measured in well 1. (Linear optimization using Legendre polynomials as basis functions.)

\begin{tabular}{|c|c|c|c|c|c|c|c|}
\hline Inversion & $\begin{array}{c}\text { Zone } \\
\text { parameter }\end{array}$ & $\begin{array}{l}\text { Initial } \\
\text { value }\end{array}$ & $\begin{array}{l}\text { Estimated } \\
\text { value }\end{array}$ & $\begin{array}{c}\text { Estimation } \\
\text { error }\end{array}$ & Unit & $S_{1}$ & $S_{2}$ \\
\hline \multirow[t]{14}{*}{ Separate } & $\mathrm{GR}_{\mathrm{sd}}$ & 15 & 9.42 & \pm 0.07 & API & 0.11 & 0.41 \\
\hline & $\mathrm{GR}_{\mathrm{sh}}$ & 160 & 153.4 & \pm 1.13 & API & 0.03 & 0.41 \\
\hline & $\Delta t_{\mathrm{sd}}$ & 50 & 55.2 & \pm 0.41 & $\mu \mathrm{s} / \mathrm{ft}$ & 0.03 & 0.40 \\
\hline & $\Delta t_{\mathrm{mf}}$ & 185 & 190.6 & \pm 1.40 & $\mu \mathrm{s} / \mathrm{ft}$ & 0.04 & 0.39 \\
\hline & $\Delta t_{\mathrm{sh}}$ & 95 & 102.9 & \pm 0.76 & $\mu \mathrm{s} / \mathrm{ft}$ & 0.10 & 0.40 \\
\hline & $\Delta t_{\mathrm{h}}$ & 220 & 202.3 & \pm 1.50 & $\mu \mathrm{s} / \mathrm{ft}$ & 0.04 & 0.41 \\
\hline & $c_{\mathrm{p}}$ & 1.0 & 1.08 & \pm 0.01 & - & 0.02 & 0.40 \\
\hline & $\rho_{\mathrm{sd}}$ & 2.6 & 2.65 & \pm 0.02 & $\mathrm{~g} / \mathrm{cm}^{3}$ & 0.02 & 0.41 \\
\hline & $\rho_{\mathrm{sh}}$ & 2.45 & 2.57 & \pm 0.02 & $\mathrm{~g} / \mathrm{cm}^{3}$ & 0.10 & 0.39 \\
\hline & $\Phi_{\mathrm{N}, \mathrm{mf}}$ & 1.0 & 0.83 & \pm 0.01 & $\mathrm{v} / \mathrm{v}$ & 0.02 & 0.39 \\
\hline & $\Phi_{\mathrm{N}, \mathrm{sh}}$ & 0.2 & 0.31 & \pm 0.02 & $\mathrm{v} / \mathrm{v}$ & 0.15 & 0.40 \\
\hline & $K_{\mathrm{sh}}$ & 2.5 & 3.21 & \pm 0.02 & $\%$ & 0.03 & 0.41 \\
\hline & $K_{\mathrm{mf}}$ & 1.5 & 1.82 & \pm 0.02 & $\%$ & 0.01 & 0.41 \\
\hline & $K_{\text {sd }}$ & 0.5 & 0.62 & \pm 0.01 & $\%$ & 0.01 & 0.41 \\
\hline \multirow[t]{3}{*}{ Joint I } & $\mathrm{GR}_{\mathrm{sd}}$ & 150 & 153.4 & \pm 1.13 & API & 0.03 & 0.40 \\
\hline & $\Delta t_{\mathrm{sh}}$ & 100 & 102.5 & \pm 0.86 & $\mu \mathrm{s} / \mathrm{ft}$ & 0.12 & 0.40 \\
\hline & $\Delta t_{\mathrm{sd}}$ & 50 & 55.3 & \pm 0.53 & $\mu \mathrm{s} / \mathrm{ft}$ & 0.07 & 0.44 \\
\hline \multirow[t]{2}{*}{ Joint II } & $R_{\mathrm{sh}}$ & 2.0 & 2.18 & \pm 0.05 & ohm-m & 0.08 & 0.43 \\
\hline & $R_{\mathrm{w}}$ & 0.5 & 0.58 & \pm 0.06 & ohm-m & 0.09 & 0.44 \\
\hline
\end{tabular}

efficients, whereas $S_{2}$ gives the mean correlation between the estimated zone and petrophysical (volumetric) parameters. Both of them refers to moderate correlation relations. The rest of the zone parameters in Table 1 correlates strongly to the petrophysical parameters (e.g., $S_{2}=0.76$ for $\rho_{\mathrm{h}}$ or $S_{2}=0.70$ for $S_{\mathrm{hrf}}$ ), which results in numerically unstable inversion procedure. By integrating more than one zone parameter in the same interval inversion procedure, we find several highly correlated model parameters. In the next step, by leaving the volumetric and control parameters unchanged, we seek an estimate to $\mathrm{GR}_{\mathrm{sd}}, \Delta t_{\mathrm{sh}}, \Delta t_{\mathrm{sd}}$, and $R_{\mathrm{sh}}, R_{\mathrm{w}}$ in a joint inversion procedure, respectively (joint I and joint II in Table 3). The total number of unknowns is $4(Q *+1)+3=$ 183 and $4(Q *+1)+2=182$, respectively, which slightly reduces the overdetermination ratio (approximately 6.4). We find the optimal solution after 10 iteration steps by using a zero regularization factor. The data distance and the average correlation between the unknowns have not changed considerably. The values of $S_{1}$ and $S_{2}$ still show reliable inversion results. There is a theoretical possibility to involve more zone parameters in the interval inversion procedure. In that case, we suggest the use of global optimization methods, which give a practically initial-model-independent and derivative-free solution (Dobróka and Szabó, 2011). Strongly correlated parameters should be determined out of the inversion procedure.

\section{CONCLUSIONS}

We present a novel inversion method for improving the accuracy and reliability of petrophysical parameter estimation based on well logs. The interval inversion method, with the advantages of great overdetermination and stability, is extended to inhomogeneous formations. We solve a highly overdetermined inversion procedure to give a more accurate estimate of petrophysical parameters. We use Legendre polynomials as basis functions for series expansion to increase the vertical resolution in hydrocarbon-bearing shaly sand formations. The orthonormal set of basis functions gives relatively small correlation between the model parameters, which assures a very stable inversion process and reliable estimation results. We suggest a method for selecting the adequate processing interval for interval inversion and the optimal number of inversion unknowns to make a balance between the vertical resolution (stability) and the accuracy of inversion results. The case study shows that the most accurate estimation can be given to porosity and shale volume, whereas water saturation correlated strongly with the other petrophysical parameters is less accurate. As an important quantity in hydrocarbon reserve calculation, we reduce the estimation error of water saturation (invaded zone) significantly compared with local inversion, especially in low-permeability formations. We propose the application of the interval inversion method not merely in high-porosity sandstones but also in low-permeability shaly sands or nonconventional reservoirs. 
There is a possibility to involve suitable zone parameters used as unknown to the interval inversion procedure. The estimation of zone parameters for the entire processed depth is especially useful when we do not have a priori information about the physical parameters of rock matrices and pore fluids or textural properties of the formations. This is the most important feature of interval inversion that local inversion does not have, because the latter is unfitted to determine petrophysical (such as zone parameters) or geometric properties (layer-boundary coordinates) of distant formations in one inversion procedure. This also improves the accuracy of the response functions. The selection of inversion unknowns must be based on parameter sensitivity functions and the model correlation matrix. Owing to its high overdetermination, the interval inversion method can be extended to the evaluation of multimineral (complex) reservoirs and to crosswell applications using basis functions depending on the lateral coordinates, too. The interval inversion method can be advantageously used in the evaluation of multimineral rocks because the local inverse problem normally is underdetermined and the evaluation is conventionally made by deterministic methods. However, as we have more mineral components, the number of expansion coefficients may highly increase. Therefore, we suggest using a moderate number of series expansion coefficients to maintain a high overdetermination ratio and stability of the inverse problem. The optimal number of coefficients can be determined by the simultaneous test of average model correlation and data distance. As presented in the paper, with the determination of the spatial distribution of petrophysical properties, zone parameters, and layer boundaries, well log analysts can automate fully the process of formation evaluation, which makes the interval inversion method a powerful tool in reservoir modeling.

\section{ACKNOWLEDGMENTS}

M. Dobróka as the leading researcher of OTKA project no. K109441 and N. Szabó as the leading researcher of OTKA project no. PD-109408 are grateful for the support of the Hungarian Scientific Research Fund. Special thanks go to I. V. Tóth to set forward the validation of the interval inversion procedure by providing the results of CLASS interpretation, well-to-well correlation, and core data.

\section{REFERENCES}

Alberty, M., and K. Hashmy, 1984, Application of ULTRA to log analysis: SPWLA Symposium Transactions, paper Z, 1-17.

Archie, G. E., 1942, The electrical resistivity log as an aid in determining some reservoir characteristics: Transactions of the AIME, 146, 54-62, doi: $10.2118 / 942054-G$

Baker Atlas, 1996, OPTIMA: eXpress reference manual: Baker Atlas, Western Atlas International, Inc.

Ball, S. M., D. M. Chace, and W. H. Fertl, 1987, The Well Data System (WDS): An advanced formation evaluation concept in a microcomputer environment: Proceedings of the SPE Eastern Regional Meeting, paper 17034, 61-85.

Dobróka, M., and N. P. Szabó, 2001, The inversion of well log data using simulated annealing method: Geosciences, Publications of the University of Miskolc, Series A (Mining), 59, 115-137.

Dobróka, M., and N. P. Szabó, 2011, Interval inversion of well-logging data for objective determination of textural parameters: Acta Geophysica, 59 907-934.

Dobróka, M., and N. P. Szabó, 2012, Interval inversion of well-logging data for automatic determination of formation boundaries by using a float- encoded genetic algorithm: Journal of Petroleum Science and Engineering, 86-87, 144-152, doi: 10.1016/j.petrol.2012.03.028.

Dobróka, M., N. P. Szabó, E. Cardarelli, and P. Vass, 2009, 2D inversion of borehole logging data for simultaneous determination of rock interfaces and petrophysical parameters: Acta Geodaetica et Geophysica Hungarica, 44, 459-479, doi: 10.1556/AGeod.44.2009.4.7.

Dobróka, M., N. P. Szabó, and E. Turai, 2012, Interval inversion of borehole data for petrophysical characterization of complex reservoirs: Acta Geodaetica et Geophysica Hungarica, 47, 172-184, doi: 10.1556/AGeod.47 .2012.2.6.

Dobróka, M., H. Szegedi, J. Somogyi Molnár, and P. Szúcs, 2015, On the reduced noise sensitivity of a new Fourier transformation algorithm: Mathematical Geosciences, 47, 679-697, doi: 10.1007/ s11004-014-9570-x.

Dobróka, M., and L. Völgyesi, 2008, Inversion reconstruction of gravity potential based on gravity gradients: Mathematical Geosciences, 40, 299-311, doi: 10.1007/s11004-007-9139-z.

Drahos, D., 1984, Electrical modeling of the inhomogeneous invaded zone: Geophysics, 49, 1580-1585, doi: 10.1190/1.1441566.

Geman, S., and D. Geman, 1984, Stochastic relaxation, Gibbs distributions, and the Bayesian restoration of images: IEEE Transactions on Pattern Analysis and Machine Intelligence, PAMI-6, 721-741, doi: 10.1109/ TPAMI.1984.4767596.

Gyulai, Á., and T. Ormos, 1999, A new procedure for the interpretation of VES data: 1.5-D simultaneous inversion method: Journal of Applied Geophysics, 41, 1-17, doi: 10.1016/S0926-9851(98)00034-2.

Gyulai, A., and E. E. Tolnai, 2012, 2.5D geoelectric inversion method using series expansion: Acta Geodaetica et Geophysica Hungarica, 47, 210222, doi: 10.1556/AGeod.47.2012.2.9.

Heidari, Z., C. Torres-Verdín, and W. E. Preeg, 2012, Improved estimation of mineral and fluid volumetric concentrations from well logs in thinly bedded and invaded formations: Geophysics, 77, WA79-WA98, doi 10.1190/geo2011-0454.1.

Horváth, Sz. B., 1973, The accuracy of petrophysical parameters as derived by computer processing: The Log Analyst, 14, 16-33.

Kis, M., 2002, Generalised series expansion (GSE) used in DC geoelectricseismic joint inversion: Journal of Applied Geophysics, 50, 401-416, doi: 10.1016/S0926-9851(02)00167-2.

Maiti, S., R. K. Tiwari, and H.-J. Kümpel, 2007, Neural network modelling and classification of lithofacies using well log data: A case study from KTB borehole site: Geophysical Journal International, 169, 733-746, doi: 10.1111/j.1365-246X.2007.03342.x.

Marquardt, D. W., 1959, Solution of non-linear chemical engineering models: Chemical Engineering Progress, 55, 65-70.

Mayer, C., and A. Sibbit, 1980, GLOBAL, a new approach to computerprocessed log interpretation: Proceedings of the 55th SPE Annual Fall Technical Conference and Exhibition, paper 9341, 1-14.

McCarthy, P. C., J. E. Sayre, and B. L. R. Shawyer, 1993, Generalized Legendre polynomials: Journal of Mathematical Analysis and Applications, 177, 530-537, doi: 10.1006/jmaa.1993.1275.

Mendoza, A., C. Torres-Verdín, and W. E. Preeg, 2010, Linear iterative refinement method for the rapid simulation of borehole nuclear measurements, part I: Vertical wells: Geophysics, 75, E9-E29, doi: 10.1190/1 3267877 .

Menke, W., 1984, Geophysical data analysis: Discrete inverse theory: Academic Press Inc.

Michalewicz, Z., 1996, Genetic algorithms + data structures = evolution programs: Springer-Verlag Berlin Heidelberg New York Inc.

Poupon, A., and J. Leveaux, 1971, Evaluation of water saturation in shaly formations: SPWLA 12th Annual Logging Symposium Transactions, 1-2.

Schlumberger, 1989, Log interpretation principles/applications: Seventh printing: Schlumberger Co.

Sen, M. K., and P. L. Stoffa, 2013, Global optimization methods in geophysical inversion: Cambridge University Press.

Simandoux, P., 1963, Dielectric measurements in porous media and application to shaly formation: Revue de L'Institut Français du Pétrole, 18, $193-215$.

Tang, X. M., and C. H. Cheng, 1993, Effects of a logging tool on the Stoneley waves in elastic and porous boreholes: The Log Analyst, 34, 46-56.

Turai, E., 2011, Data processing method developments using TAU-transformation of time-domain IP data II. Interpretation results of field measured data: Acta Geodaetica et Geophysica Hungarica, 46, 391-400, doi: 10 .1556/AGeod.46.2011.4.2.

Wyllie, M. R. J., A. R. Gregory, and L. W. Gardner, 1956, Elastic wave velocities in heterogeneous and porous media: Geophysics, 21, 41-70, doi: 10.1190/1.1438217. 